\title{
On the UV renormalizability of noncommutative field theories
}

\author{
Swarnendu Sarkar \\ The Institute of Mathematical Sciences, \\ CIT Campus, Chennai - 600 113, INDIA \\ swarnen@imsc.ernet.in
}

\begin{abstract}
UV/IR mixing is one of the most important features of noncommutative field theories. As a consequence of this coupling of the UV and IR sectors, the configuration of fields at the zero momentum limit in these theories is a very singular configuration. We show that the renormalization conditions set at a particular momentum configuration with a fixed number of zero momenta, renormalizes the Green's functions for any general momenta only when this configuration has same set of zero momenta. Therefore only when renormalization conditions are set at a point where all the external momenta are nonzero, the quantum theory is renormalizable for all values of nonzero momentum. This arises as a result of different scaling behaviors of Green's functions with respect to the UV cutoff $(\Lambda)$ for configurations containing different set of zero momenta. We study this in the noncommutative $\phi^{4}$ theory and analyse similar results for the Gross-Neveu model at one loop level. We next show this general feature using Wilsonian RG of Polchinski in the globally $O(N)$ symmetric scalar theory and prove the renormalizability of the theory to all orders with an infrared cutoff. In the context of spontaneous symmetry breaking (SSB) in noncommutative scalar theory, it is essential to note the different scaling behaviors of Green's functions with respect to $\Lambda$ for different set of zero momenta configurations. We show that in the broken phase of the theory the Ward identities are satisfied to all orders only when one keeps an infrared regulator by shifting to a nonconstant vacuum.
\end{abstract}




\section{Contents}

1. Introduction 1

2. UV/IR mixing and UV renormalizability 3

3. The noncommutative Gross-Neveu model 7

4. SSB in Non-commutative scalar theory 8

4.1 One loop analysis 9

4.2 Renormalizability to all orders : Review of The commutative case 11

4.3 The noncommutative case 16

5. Conclusion 23

A. RG equations for $L, B$ and $V$

\section{Introduction}

Noncommutative spacetimes and field theories defined on them have been studied extensively for the past few years mainly motivated from string theory [1, 2, 3]. Apart from this fact that these theories arise as low energy limits of string theory in a constant $B_{\mu \nu}$ background, their study as field theories in their own right is quite facinating. For reviews on the subject see [ [ , 5, 6]. Various interesting aspects of these theories have been studied rigorously recently [7]-[31]. The most important of these being the intriguing mixing of UV and IR divergences which is a direct consequence of the noncommutavity of the background spacetime. Perturbative studies of these field theories carried out extensively revealed various nontrivial aspects arising from this transmutation of UV into IR divergences . One of the most important being the alteration of the conventional Wilsonian picture of renormalization group flows in the very low momentum domain. The UV renormalizability of these theories has been argued [8] and in some cases expiicitly shown upto two loops [19. The $\lambda \phi^{4}$ theory has been shown to be renormalizable as long as the external momenta, $p$ for the $n$-point functions are such that $\Lambda^{2}$ pop $>1$. [17. However given that we are only interested in the continuum limit, $\Lambda \rightarrow \infty$, the inequality is not satisfied only when $p$ is restricted to the zero momentum value. 
The possibility that these theories would ultimately be defined with an infrared cutoff still exists. It was shown [16] that phase transitions if possible can only occur at a finite momentum leading to a nonhomogeneous phase.

Normally in commutative field theories the renormalization conditions required to absorb the infinities in the Green's functions of a particular configuration of fields, with momenta around some scale, leads to the infinities being absorbed from the Green's functions at all scales i.e. the functional dependence of the divergent $n$-point functions on the UV cutoff $\Lambda_{0}$ is same at all values of external momenta. On the other hand, in noncommutative theories, due to coupling of the UV and IR sectors, the zero momentum limit of a particular configuration of fields is singular. We show that Greens's functions scale with different coefficients of $\Lambda$ for configurations which differ by the number of zero external momenta. If the renormalization conditions are set at a point where all the external momenta are nonzero, then the bare couplings defined through this would have a different $\Lambda_{0}$ dependence from the case when the renormalization point consists of a number of zero momenta. This shows that with the former renormalization condition, the theory for all values of external momenta, $p \neq 0$ is renormalizable, while the latter leads to a nonrenormalizable theory. We discuss this issue in the noncommutative $\phi^{4}$ theory as well as in the Gross-Neveu model as shown in 11.

We next study the renormalizability of the globally $O(N)$ symmetric noncommutative scalar theory in its symmetric and its broken phases to all orders. First we review the same for the commutative case in the renormalization group approach. 32 33. The noncommutative theory is then proved to be renormalizable to all orders with an infrared cutoff. Keeping in mind the observations stated in the previous paragraph, we seperate the sector with external momenta such that $\Lambda^{2} p o p>1$ from that of $\Lambda^{2}$ pop $<1$. In the latter case, since in the continuum limit $p$ is only restricted to zero, the full quantum theory for nonzero $p$ will thus be defined with an Infrared cutoff. Because of the presence of the infrared cutoff for the external momenta, we shall also formally introduce an IR cutoff for the internal loop momenta $\left(\Lambda_{I R}\right)$. However we shall see that the in the loop computations the IR cutoff for the internal momenta is not necessary as there are no IR divergences in the loop intergals in this $\mathrm{RG}$ approach. IR divergences appear in the continuum limit as the zero momentum field configuration is approached from a nonzero momentum field configuration. Ofcourse with an IR cutoff in the external momenta, $p$ such that, $\Lambda^{2} p o p>1$, there are no IR divergences in the theory. However IR divergences do appear in perturbation theory. This is illustrated by an example of a 2-point diagram computation.

It may seem that $\left(\Lambda_{I R}\right)$ may be needed, so that the the cannonical scaling of the relevant and irrelevant operators are not affected by the UV/IR transmutation. However this is not the case. This point will be clarified in section 4 .

We then demonstrate how the different scaling behaviors of the Green's functions for configurations having different set of zero momenta plays a crucial role when 
proving the renormalizability of the spontaneously broken phase. It was shown in [15] that the renormalizability of the broken symmetric phase to one loop could be proved by shifting to a phase where the vacuum was a nonconstant background field. This nonconstant vacuum acts as an infrared regulator.

This paper is organised as follows. In section 2 we study the noncommutative $\phi^{4}$ theory and show that the UV cutoff $(\Lambda)$ dependence of the bare couplings are different depending on whether we set the renormalization conditions at $p=0$ or $p \neq 0$. In section 3 we analyse some more results for the noncommutative Gross-Neveu model along the same line as section 2. In section 4 we study SSB in noncommutative scalar theory with global $O(N)$ symmetry in its symmetric phase. We first review the one loop results from [12, 15]. Next we prove the renormalizability of the symmetric phase of the globally $O(N)$ symmetric noncommutative scalar theory to all orders, after reviewing the same for the commutative case. For the broken phase we demonstrate that, by going to a phase which is translationally noninvariant i.e. by keeping the shift $v$ as a nonconstant background field one is able to work with a infrared regulator so that the problem of different scaling behaviors of Green's functions for nonzero external momenta from that of the zero momentum case does not arise. With this infrared regulator we prove the renormalizability of the broken phase of the theory to all orders. We give our conclusions in section 5 .

\section{UV/IR mixing and UV renormalizability}

In this section we show that the one loop Green's functions defines two different renormalization conditions depending on whether we set $p=0$ right in the begining or approach this limit with a nonsingular, $p \neq 0$ configuration. We study the noncommutative $\phi^{4}$ theory to show this. For an introduction to noncommutative scalar theory see [8].

The lagrangian for the theory is,

$$
\mathcal{L}_{E}=-\left[\frac{1}{2}\left(\partial_{\mu} \phi\right)^{2}+\frac{1}{2} m^{2} \phi^{2}+\frac{\lambda}{4} \phi * \phi * \phi * \phi\right]
$$

where,

$$
\phi_{1} * \phi_{2}=\left.e^{\frac{i}{2} \partial_{\mu}^{y} \theta^{\mu \nu} \partial_{\nu}^{z}} \phi_{1}(y) \phi_{2}(z)\right|_{y=z=x}
$$

and $\theta$ is an antisymmetric matrix. The propagator for the theory, is same as that

of the commutative theory. Only the interaction term has a nontrivial momentum dependence. The inteaction vertex is given by,

$$
\begin{aligned}
-2 \lambda V(\mathbf{p})=-2 \lambda\left[\cos \left(p_{1} \wedge p_{2}\right) \cos \left(p_{3} \wedge p_{4}\right)\right. & +\cos \left(p_{1} \wedge p_{3}\right) \cos \left(p_{2} \wedge p_{4}\right) \\
& \left.+\cos \left(p_{1} \wedge p_{4}\right) \cos \left(p_{2} \wedge p_{3}\right)\right]
\end{aligned}
$$


The only divergent functions for the theory are the two point and the four point functions. The diagrams corresponding to the one loop contributions to these functions are shown in figures 1 and 2 .

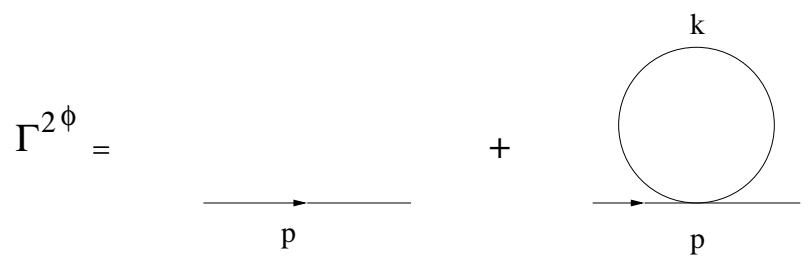

Figure 1:

$$
\Gamma^{2}=-\left[p^{2}+m^{2}\right]-\lambda \int \frac{d^{4} k}{(2 \pi)^{4}} \frac{2+\cos (p \wedge k)}{k^{2}+m^{2}}
$$

The cos term inside the integral regulates the second part of the integral and is finite for $p \neq 0$.

$$
\begin{aligned}
\Gamma^{2}=- & {\left[p^{2}+m^{2}\right]-\frac{\lambda}{8 \pi^{2}}\left[\Lambda^{2}-m^{2} \ln \left(\frac{\Lambda^{2}}{m^{2}}\right)+O(1)\right] } \\
- & \frac{\lambda}{16 \pi^{2}}\left[\Lambda_{e f f}^{2}(p)-m^{2} \ln \left(\frac{\Lambda_{e f f}^{2}(p)}{m^{2}}\right)+O(1)\right]
\end{aligned}
$$

where,

$$
\begin{aligned}
\Lambda_{e f f}^{2}(p) & =\frac{1}{\frac{1}{\Lambda^{2}}+p o p} \\
p o p & =-\frac{p^{\mu} \theta_{\mu \nu}^{2} p^{\nu}}{4}
\end{aligned}
$$

$\Lambda$ is the UV cutoff. We shall call terms containing $\Lambda_{\text {eff }}^{2}(p)$ as nonplanar terms.

$$
\Gamma^{4}=-2 \lambda V(\mathbf{p})+2 \lambda^{2} \int \frac{d^{4} k}{(2 \pi)^{4}} \frac{F\left(p_{1}, p_{2}, p_{3}, p_{4}, p, k\right)}{\left(k^{2}+m^{2}\right)\left[(p-k)^{2}+m^{2}\right]}+\mathrm{s} \text { and u channels (2.7) }
$$

where $F\left(p_{1}, p_{2}, p_{3}, p_{4}, p, k\right)$ is a function of terms containing cos of the external momenta.

$$
\Gamma^{4}=-2 \lambda V(\mathbf{p})+4 \lambda^{2} V(\mathbf{p}) \int \frac{d^{4} k}{(2 \pi)^{4}} \frac{1}{\left(k^{2}+m^{2}\right)\left[(p-k)^{2}+m^{2}\right]}+N P
$$




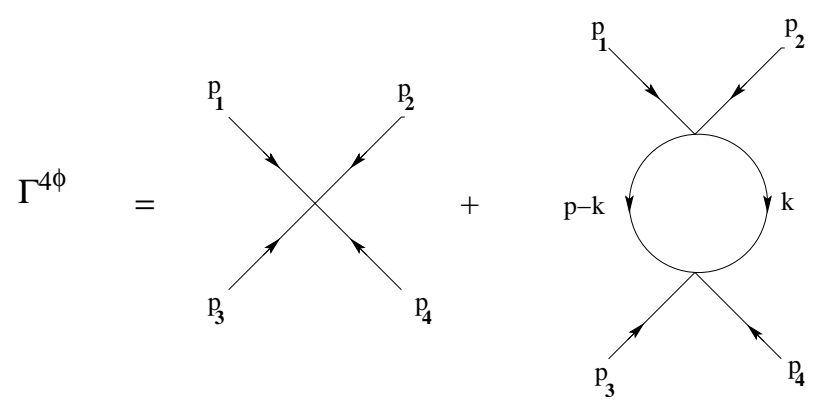

Figure 2:

where $N P$ are the Nonplanar terms. These terms would give rise to IR divergences as the external momenta goes to zero. The UV divergent piece for this four point amplitude is given by,

$$
\Gamma^{4} \sim \frac{\lambda^{2}}{4 \pi^{2}} V(\mathbf{p}) \ln \left(\frac{\Lambda^{2}}{m^{2}}\right)
$$

The two and the four point contributions to the effective action to one loop is,

$$
\begin{aligned}
-S_{e f f} & =\frac{1}{2 !} \int d p \phi(p) \phi(-p)\left[p^{2}+m^{2}+\frac{\lambda}{8 \pi^{2}} \Lambda^{2}-\frac{\lambda}{8 \pi^{2}} m^{2} \ln \left(\frac{\Lambda^{2}}{m^{2}}\right)+N P\right] \\
& +\frac{1}{4 !} \int d p_{1} d p_{2} d p_{3} d p_{4}[P+N P] \phi\left(p_{1}\right) \phi\left(p_{2}\right) \phi\left(p_{3}\right) \phi\left(p_{4}\right) \delta\left(\sum p_{i}\right)
\end{aligned}
$$

where, $P$ is the planar term from the four point amplitude given by,

$$
P=V(\mathbf{p})\left\{2 \lambda-\frac{\lambda^{2}}{4 \pi^{2}} \ln \left(\frac{\Lambda^{2}}{m^{2}}\right)\right\}
$$

The renormalised parameters may now be defined as,

$$
\begin{aligned}
m_{R}^{2} & =m^{2}+\frac{\lambda}{8 \pi^{2}}\left[\Lambda^{2}-m^{2} \ln \left(\frac{\Lambda^{2}}{m^{2}}\right)\right] \\
\lambda_{R} & =\lambda-\frac{\lambda^{2}}{8 \pi^{2}} \ln \left(\frac{\Lambda^{2}}{m^{2}}\right)
\end{aligned}
$$

As the zero momentum limit is approached, the $N P$ terms in equation (2.7) give rise to $I R$ divergences, however the renormalization conditions in equation (2.12) lead to a UV renormalizable quantum theory at one loop.

Now let us consider the case where the effective action at the zero momentum field configuration is defined by [34], 


$$
V_{e f f}=\sum_{n=1}^{\infty} \frac{1}{n !} \Gamma^{n}(0,0, \ldots, 0) \phi^{n}
$$

We use this to define $\lambda(\Lambda)$. In this case at one loop level there are no nonplanar diagrams and the potential to one loop is exactly equal to the commutative theory. The external momenta are all put to zero before all loop calculations. To one loop the effective potential is given by,

$$
\begin{aligned}
V_{e f f} & =\frac{1}{2} m^{2} \phi^{2}+\frac{\lambda}{4} \phi^{4}+\frac{1}{2} \int^{\Lambda} \frac{d^{4} k}{(2 \pi)^{4}} \ln \left(1+\frac{3 \lambda \phi^{2}}{k^{2}+m^{2}}\right) \\
& =\frac{1}{2} m^{2} \phi^{2}+\frac{\lambda}{4} \phi^{4}+\frac{3 \lambda \phi^{2}}{32 \pi^{2}}\left[\Lambda^{2}-m^{2} \ln \left(\frac{\Lambda^{2}}{m^{2}}\right)\right]+\frac{9 \lambda^{2}}{64 \pi^{2}} \phi^{4} \ln \left(\frac{3 \lambda \phi^{2}}{\Lambda^{2}}\right)
\end{aligned}
$$

where in the final expression we have dropped terms which have negative powers or are independent of $\Lambda$.

The renormalized quantities would now be defined by,

$$
\begin{gathered}
\left.\frac{d^{2} V}{d \phi^{2}}\right|_{\phi=0}=m_{R}^{2} \\
\left.\frac{d^{4} V}{d \phi^{4}}\right|_{\phi=\phi_{0}}=6 \lambda_{R}
\end{gathered}
$$

These lead to,

$$
\begin{aligned}
m_{R}^{2} & =m^{2}+\frac{3 \lambda}{16 \pi^{2}}\left[\Lambda^{2}-m^{2} \ln \left(\frac{\Lambda^{2}}{m^{2}}\right)\right] \\
\lambda_{R} & =\lambda-\frac{9 \lambda^{2}}{16 \pi^{2}} \ln \left(\frac{\Lambda^{2}}{3 \phi_{0}^{2}}\right)
\end{aligned}
$$

From equations (2.12) and (2.18) it is clear that the renormalization conditions defined by equation (2.18) would not lead to a renormalizable noncommutative theory for non zero external momenta. As noted before, the configuration of fields with $p=0$ is singular and the two different renormalization conditions (2.12), 2.18) occur as a consequence of setting $p=0$ right in the begining or of approaching this configuration as a limit $p \rightarrow 0$. This is a generic feature of noncommutative theories. The origin of this is the transmutation of UV divergences into the IR divergences.

In terms of the renormalization group flows, equations (2.12), (2.18) states that at the one loop level the relevant (relevant plus marginal) coupling $\lambda$ scales with respect to $\Lambda$ with different coefficients. This means that the functional dependence of the bare coupling on the UV cutoff $\Lambda$ are different in the two cases. We shall see this in section 4 , where the relevant couplings would scale with different coefficients of $\Lambda$ depending on whether or not the external momenta are such that $\Lambda^{2} p o p<<1$. 


\section{The noncommutative Gross-Neveu model}

In this section we review some of the results of the noncommutative Gross-Neveu model [11] which are along the same line as those of the previous section.

The lagrangian for the noncommutative Gross-Neveu model is,

$$
\mathcal{L}_{E}=-\left[\frac{1}{2} \bar{\psi}^{i} \gamma^{\mu} \partial_{\mu} \psi^{i}+\frac{\lambda}{8 N} \bar{\psi}^{i} * \psi^{i} * \bar{\psi}^{j} * \psi^{j}\right]
$$

Where $\psi$ is a 2 -component spinor and $\gamma^{\mu}$ are $2 \times 2$ Dirac matrices. To evaluate the large $N$ limit of the effective action it is helpful to introduce an auxillary field $\sigma$, so that,

$$
\mathcal{L}_{E}=-\left[\frac{1}{2} \bar{\psi}^{i} \gamma^{\mu} \partial_{\mu} \psi^{i}-\frac{8 N}{\lambda} \sigma^{2}-2 \sigma * \bar{\psi}^{i} * \psi^{i}\right]
$$

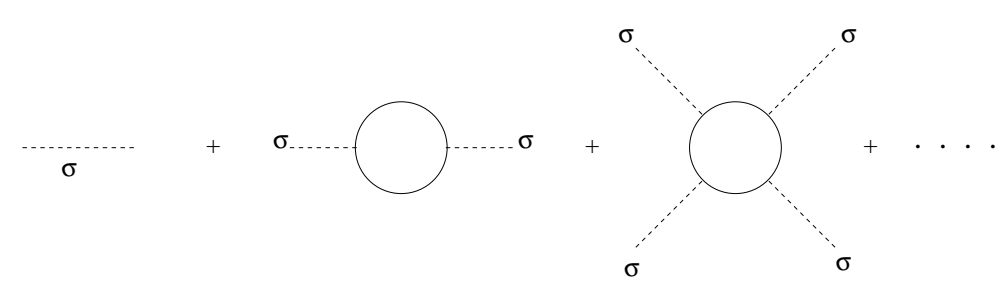

Figure 3:

We now evaluate the effective action at the zero momentum field configuration as defined by equation (2.13). The processes with only $\sigma$ field on the external legs would contribute to the lowest order in $\frac{1}{N}$ [35]. These diagrams contributing to this lowest order are shown in figure 3 .

$$
\begin{aligned}
V_{e f f} & =\frac{8 N}{\lambda} \sigma^{2}-N \sum_{n=1}^{\infty} \int \frac{d^{2} k}{(2 \pi)^{2}} \frac{1}{2 n}\left(\frac{4 \sigma^{2}}{k^{2}}\right)^{n} \\
& =\frac{8 N}{\lambda} \sigma^{2}-\frac{N \sigma^{2}}{2 \pi}\left[\ln \left(\frac{\Lambda^{2}}{4 \sigma^{2}}\right)+O(1)\right]
\end{aligned}
$$

The renormalization condition,

$$
\left.\frac{\partial^{2} V_{e f f}}{\partial \sigma^{2}}\right|_{\sigma^{2}=\sigma_{0}^{2}}=1
$$

defines the renormalized coupling $\lambda_{R}$ as,

$$
\frac{1}{\lambda_{R}}=\frac{1}{\lambda}-\frac{1}{16 \pi} \ln \left(\frac{\Lambda^{2}}{4 \sigma_{0}^{2}}\right)
$$


We now write down the effective action for nonzero external momenta in the large $N$ limit. Only the two point function is divergent. To extract the renormalization condition it is sufficient to evaluate the effective action with contributions upto the two point function only.

$$
S_{e f f}=\frac{1}{2} \int d^{2} p \sigma(p) \Gamma^{\sigma \sigma} \sigma(-p)
$$

where,

$$
\Gamma^{\sigma \sigma}=\frac{8 N}{\lambda}-N \int \frac{d^{2} k}{(2 \pi)^{2}} \frac{k \cdot(p+k)}{k^{2}(k+p)^{2}}+N P
$$

$N P$ is the nonplanar term. The renormalized coupling will now be defined as,

$$
\frac{1}{\lambda_{R}}=\frac{1}{\lambda}-\frac{1}{32 \pi} \ln \left(\frac{\Lambda^{2}}{p^{2}}\right)
$$

The renormalization condition, equation (3.9) gives a UV renormalizable quantum theory for any nonzero value of external momenta, similar to the results obtained in the previous section. If one defines the renormalized coupling as equation (3.6), the quantum theory at nonzero external momenta is nonrenormalizable. These two renormalization conditions correspond to two different bare theories as argued at the end of section 2 .

The ground state of the theory will be defined by a particular configuration of fields. If it is defined with zero external momenta, we have noted that this is a very singular field configuration and Green's functions in this configuration has a different $\Lambda$ dependence from the nonzero momenta field configurations. If the renormalization conditions are set at zero momentum the theory is nonrenormalizable. In the next section we shall see how this plays a crucial role in deciding the renormalizability of the Broken Phase in a spontaneously broken global symmetric theory.

\section{SSB in Non-commutative scalar theory}

In the previous two sections we have seen that due to the singular behavior of the IR limit in nncommutative field theories, the Green's functions have different $\Lambda$ dependences for nonzero and zero momentum field configurations. In this section we show how this plays a crucial role when proving renormalizability of the broken phase of a spontaneously broken globally $O(N)$ symmetric theory. We first review

our one loop results [15] and then in the later part of this section we shall prove following [33], the renormalizability of the symmetric phase as long as the external 
momenta $p \neq 0$ such that $\Lambda^{2} p o p>1$. Formally with an IR cutoff in the external momenta, one should also introduce an IR cutoff for the internal loop momenta $\left(\Lambda_{I R}\right)$. The presence of this IR cutoff in the loop computations would be implicitly assumed. This point is elaborated in section 4.2. The relevant couplings for the zero momentum configuration of fields scale with different coefficients of the UV cutoff from the $p \neq 0$ configurations. If the renormalization conditions are set in these two momentum regimes, the bare couplings will have different $\Lambda$ dependences. At the end of the section we shall show the renormalizability of the broken phase of the theory to all orders in terms of the renormalization group.

\subsection{One loop analysis}

We consider a scalar field theory with global $O(2)$ symmetry in its symmetric phase in 4 dimensions. We refer the reader to [15] for the details of calculations.

The lagrangian for the theory is ${ }^{1}$,

$$
\begin{aligned}
& L_{S}=-\left[\frac{1}{2}\left(\partial_{\mu} \phi^{i}\right)^{2}-\frac{1}{2} \mu^{2}\left(\phi^{i}\right)^{2}+\frac{\lambda}{4} \phi^{i} * \phi^{i} * \phi^{j} * \phi^{j}\right] \\
& i, j=1,2
\end{aligned}
$$

Here $\mu^{2}>0$, so that at the tree-level the theory undergoes SSB. The global $\mathrm{O}(2)$ symmetry of the quantum theory implies that the green's functions satisfy the following set of Ward identities in the symmetric phase,

$$
\begin{aligned}
& \left.\frac{\delta^{2} \Gamma}{\delta \phi_{1}^{2}}\right|_{\phi_{1}=\phi_{2}=0}=\left.\frac{\delta^{2} \Gamma}{\delta \phi_{2}^{2}}\right|_{\phi_{1}=\phi_{2}=0} \\
& \left.\frac{\delta^{4} \Gamma}{\delta \phi_{1}^{4}}\right|_{\phi_{1}=\phi_{2}=0}=\left.3 \frac{\delta^{4} \Gamma}{\delta \phi_{1}^{2} \delta \phi_{2}^{2}}\right|_{\phi_{1}=\phi_{2}=0}
\end{aligned}
$$

and in the broken phase which is defined by shifting the fields, $\phi_{1}=\sigma+v$ and $\phi_{2}=\pi$,

$$
\left.v \frac{\delta^{2} \Gamma}{\delta \pi^{2}}\right|_{\sigma=\pi=0}=\left.\frac{\delta \Gamma}{\delta \sigma}\right|_{\sigma=\pi=0}
$$

where the $\phi_{1}$ field has been shifted by a constant amount $v$ which fixes the vacuum for the broken phase.

\footnotetext{
${ }^{1}$ Note that in the noncommutative theory, there are various possible inequivalent orderings of the fields for the quartic term. One of these terms has been chosen as an example. The proof of the renormalizability of the globally $O(N)$ symmetric theory, in its symmetric as well as in its broken phases, to all orders, outlined in the latter part of this section remains unaltered for any such $O(N)$ symmetric quartic term.
} 
The set of Ward identities, equation (4.2), can be verified to one loop [15], showing that the quantum theory is symmetric. However care must be taken in defining the broken phase so that equation (4.3) holds.

If one naively shifts to a translationally invariant vacuum, the Ward identity, equation (4.3) only holds when the order of the continuum and the IR limits is such that the IR divergences on the RHS of (4.3) are transmuted to UV divergences i.e. the vacuum is defined with $p=0$ before all loop calculations. It is important to note here that shifting fields by a constant amount $v$ at the tree-level itself causes the $\sigma$-tadpole amplitude to scale with respect to $\Lambda$ in the same way as the second case in section 2 . Where as the $\Gamma^{\pi \pi}$ amplitude has the same $\Lambda$ dependence when the external momenta are put to zero before all loop computations. This makes the broken phase nonrenormalizable with the same number of counterterms as the symmetric phase. This point will be elaborated at the end of this section. However in order to define a UV renormalizable theory for all values of $p$ we must work with an infrared cutoff and remove the cutoff only after all loop computations as the former case in section 2. To do so one has to shift the fields by $v$ which is not a constant. $v$ may be set to a constant after all loop calculations. This would mean that the singular field configuration, defined as the vacuum, is approached as a limit. One can also leave $v$ as a nonconstant leading to a translationally noninvariant vacuum. This later case was studied in [16] where phase transitions when a finite number of momentum modes condense is studied. In the present paper a nonconstant $v$ acts as an infrared regulator and help us to avoid proplems as noted earlier.

The Ward identity in the case where $v$ is not a constant will now be written as,

$$
\left.\int \frac{d^{4} p}{(2 \pi)^{4}} v(-p) \frac{\delta^{2} \Gamma}{\delta \pi\left(p_{1}\right) \delta \pi(p)}\right|_{\sigma=\pi=0}=\left.\frac{\delta \Gamma}{\delta \sigma\left(p_{1}\right)}\right|_{\sigma=\pi=0}
$$

Explicit computations in this case shows that,

$$
\begin{aligned}
& \Gamma^{\sigma}=v_{0} \mu^{2}-\lambda v_{0}^{3}+v_{0}\left[-3 \lambda\left(\Lambda^{2}+\mu^{2} \ln \left(\frac{\Lambda^{2}}{-\mu^{2}}\right)\right)+I R+F\right]+ \\
&+ v_{0}^{3}\left[\frac{5}{2} \ln \left(\frac{\Lambda^{2}}{-\mu^{2}}\right)+I R+F\right] \\
& \Gamma^{\pi \pi}=-p^{2}+\mu^{2}-\lambda v_{0}^{2}+\left[-3 \lambda\left(\Lambda^{2}+\mu^{2} \ln \left(\frac{\Lambda^{2}}{-\mu^{2}}\right)\right)+I R+F\right]+ \\
&+v_{0}^{2}\left[\frac{5}{2} \ln \left(\frac{\Lambda^{2}}{-\mu^{2}}\right)+I R+F\right]
\end{aligned}
$$

Where IR are the infrared divergent terms and $\mathrm{F}$ are the finite terms. It can be seen that the UV divergence structure of the two amplitudes are exactly as the Ward identity (4.3). The IR divergences appear when we go to a translationally invariant vacuum i.e. $v$ is set to a constant $v_{0}$ after all loop calculations. 


\subsection{Renormalizability to all orders : Review of The commutative case}

We now consider the commutative globally symmetric $O(N)$ scalar theory in its symmetric phase and review its renormalizability to all orders following Polchinski [33]. We discuss here the set up of the RG equations and merely state the results, which will be necessary for the later parts of this section. The reader may refer to [33] for more elaborate details and proofs.

$$
\begin{array}{r}
S(\phi)=\int \frac{d^{4} p}{(2 \pi)^{4}}\left[-\frac{1}{2} \phi^{\alpha}(p) \phi^{\alpha}(-p)\left(p^{2}-\mu^{2}\right) K^{-1}\left(\frac{p^{2}}{\Lambda^{2}}\right)\right]+L_{i n t}(\phi) \\
L_{i n t}(\phi)=\int d^{4} x\left[-\frac{1}{2} \rho_{1}^{0}\left(\phi^{\alpha}(x)\right)^{2}-\frac{1}{2} \rho_{2}^{0}\left(\partial_{\mu} \phi^{\alpha}(x)\right)^{2}-\frac{1}{4} \rho_{3}^{0}\left(\phi(x)^{\alpha} \phi(x)^{\alpha}\right)^{2}\right]
\end{array}
$$

where, $K\left(\frac{p^{2}}{\Lambda^{2}}\right)$ has a value of 1 for $p^{2}<\Lambda^{2}$ and vanishes rapidly at infinity. $\rho_{a}^{0}$ are the bare couplings defined at an UV cutoff scale $\Lambda_{0}$.

The generating functional for the theory with the cutoff, $\Lambda$ may be written as

$$
\begin{aligned}
& Z[J, \Lambda]=\int \mathcal{D} \phi \exp \left[\int \frac { d ^ { 4 } p } { ( 2 \pi ) ^ { 4 } } \left[-\frac{1}{2} \phi^{\alpha}(p) \phi^{\alpha}(-p)\left(p^{2}-\mu^{2}\right) K^{-1}\left(\frac{p^{2}}{\Lambda^{2}}\right)\right.\right. \\
& \left.\left.+J^{\alpha}(p) \phi^{\alpha}(-p)\right]+L_{i n t}(\phi)\right] \\
& \Lambda \frac{d Z[J, \Lambda]}{d \Lambda}=\int \mathcal{D} \phi\left[\int \frac { d ^ { 4 } p } { ( 2 \pi ) ^ { 4 } } \left[-\frac{1}{2} \phi^{\alpha}(p) \phi^{\alpha}(-p)\left(p^{2}-\mu^{2}\right) \Lambda \frac{\partial K^{-1}}{\partial \Lambda}\left(\frac{p^{2}}{\Lambda^{2}}\right)\right.\right. \\
& \left.\left.+J^{\alpha}(p) \phi^{\alpha}(-p)\right]+\Lambda \frac{\partial L_{i n t}(\phi)}{\partial \Lambda}\right] \exp (S(\phi))
\end{aligned}
$$

The RHS of equation (4.9) vanishes if $L$ varies as,

$$
\begin{aligned}
\Lambda \frac{\partial L}{\partial \Lambda}= & -\frac{1}{2} \int d^{4} p(2 \pi)^{4}\left(p^{2}-\mu^{2}\right)^{-1} \Lambda \frac{\partial K}{\partial \Lambda} \\
& {\left[\frac{\partial L}{\partial \phi^{\alpha}(-p)} \frac{\partial L}{\partial \phi^{\alpha}(p)}+\frac{\partial^{2} L}{\partial \phi^{\alpha}(-p) \partial \phi^{\alpha}(p)}\right] }
\end{aligned}
$$

$L$ can now be expanded in terms of its Fourier modes. The global $O(N)$ symmetry of the quantum theory implies that we can arrange the expansion as follows.

$$
L=\sum_{n=1}^{\infty} \frac{1}{2 n !} \int \prod_{i=1}^{n} \prod_{j=n+1}^{2 n} d^{4} p_{i} d^{4} p_{j} \phi^{\alpha}\left(p_{i}\right) \phi^{\alpha}\left(p_{j}\right) L_{2 n}\left(p_{1} \ldots p_{2 n}, \Lambda\right) \delta^{4}\left(\sum_{i, j} p_{i}+p_{j}\right)
$$


Define the relevant operators as,

$$
\begin{array}{r}
\rho_{1}(\Lambda)=-\left.L_{2}(p,-p, \Lambda)\right|_{p^{2}=p_{0}^{2}} \\
\rho_{2}(\Lambda)=-\left.\frac{\partial^{2}}{\partial p^{2}} L_{2}(p,-p, \Lambda)\right|_{p^{2}=p_{0}^{2}} \\
\rho_{3}(\Lambda)=-\left.L_{4}\left(p_{1}, p_{2}, p_{3}, p_{4}, \Lambda\right)\right|_{p=\bar{p}}
\end{array}
$$

Now, construct $V(\Lambda)$ such that,

$$
V(\Lambda)=\Lambda_{0} \frac{\partial L(\Lambda)}{\partial \Lambda_{0}}-\sum_{a, b} \frac{\partial L(\Lambda)}{\partial \rho_{a}^{0}} \frac{\partial \rho_{a}^{0}}{\partial \rho_{b}(\Lambda)} \Lambda_{0} \frac{\partial \rho_{b}(\Lambda)}{\partial \Lambda_{0}}
$$

where, $a, b$ runs from 1 to 3 . To prove that the theory is renormalizable or in other words to show that, for the low energy theory to be finite one has to tune a finite number of parameters, $V(\Lambda)$ must be shown to vanish at the low energy limit, $\Lambda / \Lambda_{0} \rightarrow 0$.

Expanding the following quantities similar to equation (4.11),

$$
\begin{aligned}
\frac{\partial L(\Lambda)}{\partial \rho_{b}(\Lambda)} & =\sum_{a} \frac{\partial L(\Lambda)}{\partial \rho_{a}^{0}} \frac{\partial \rho_{a}^{0}}{\partial \rho_{b}(\Lambda)} \\
& =\sum_{n=1}^{\infty} \frac{\Lambda^{4-2 n-2 \delta_{b 1}}}{2 n !} \int \frac{\prod_{i=1}^{n} \prod_{j=n+1}^{2 n} d^{4} p_{i} d^{4} p_{j}}{(2 \pi)^{8 n-4}} \phi^{\alpha}\left(p_{i}\right) \phi^{\alpha}\left(p_{j}\right) \times \\
& \times B_{b, 2 n}\left(p_{1} \ldots p_{2 n}, \Lambda\right) \delta^{4}\left(\sum_{i, j} p_{i}+p_{j}\right) \\
V(\Lambda) & =\sum_{n=1}^{\infty} \frac{\Lambda^{4-2 n}}{2 n !} \int \frac{\prod_{i=1}^{n} \prod_{j=n+1}^{2 n} d^{4} p_{i} d^{4} p_{j}}{(2 \pi)^{8 n-4}} \phi^{\alpha}\left(p_{i}\right) \phi^{\alpha}\left(p_{j}\right) \times \\
& \times V_{2 n}\left(p_{1} \ldots p_{2 n}, \Lambda\right) \delta^{4}\left(\sum_{i, j} p_{i}+p_{j}\right)
\end{aligned}
$$

Now defining,

$$
Q\left(p, \Lambda, \mu^{2}\right)=\frac{1}{\left(p^{2}-\mu^{2}\right)} \Lambda^{3} \frac{\partial K\left(\frac{p^{2}}{\Lambda^{2}}\right)}{\partial \Lambda}
$$

one arrives at the RG equations for $L, B, V$, shown in the appendix, equations (A.1), (A.3), A.5) where,

$$
\left\|f\left(p_{1}, \ldots, p_{2 n}, \Lambda\right)\right\|=\max _{p_{i}^{2}<c \Lambda^{2}}\left|f\left(p_{1}, \ldots, p_{2 n}, \Lambda\right)\right|
$$

so that, 


$$
\left\|\int \frac{d^{4} p}{(2 \pi)^{4}} Q(p, \Lambda) L_{2 n}\left(p_{1}, \ldots, p_{2 n-2}, p,-p, \Lambda\right)\right\|<C \Lambda^{4}\left\|L_{2 n}(\Lambda)\right\|
$$

where, $c$ and $C$ are constants independent of $\Lambda$. Now define the following initial conditions for the couplings,

$$
\begin{aligned}
& \rho_{1}\left(\Lambda_{R}, \Lambda_{0}, \rho^{0}\right)=0 \\
& \rho_{2}\left(\Lambda_{R}, \Lambda_{0}, \rho^{0}\right)=0 \\
& \rho_{3}\left(\Lambda_{R}, \Lambda_{0}, \rho^{0}\right)=6 \lambda_{R}
\end{aligned}
$$

Note that because of the $O(N)$ symmetry of the quantum theory it is sufficient to set boundary conditions as above. The irrelevant couplings are set to vanish at $\Lambda_{0}$. Perturbative renormalizability now means that order by order in $\lambda_{R}$ the following limit exists,

$$
\lim _{\Lambda_{0} \rightarrow \infty} \bar{L}\left(\phi, \Lambda_{R}, \lambda_{R}, \Lambda_{0}\right)=\bar{L}\left(\phi, \Lambda_{R}, \lambda_{R}, \infty\right)
$$

where,

$$
\bar{L}\left(\phi, \Lambda_{R}, \lambda_{R}, \Lambda_{0}\right)=L\left(\phi, \Lambda_{R}, \Lambda_{0}, \rho^{0}\left(\Lambda_{R}, \lambda_{R}, \Lambda_{0}\right)\right)
$$

and specifically to $r$ th order in $\lambda_{R}$,

$$
\begin{aligned}
\| \bar{L}_{2 n}^{(r)}\left(\Lambda_{R}, \Lambda_{0}\right)-\bar{L}_{2 n}^{(r)}\left(\Lambda_{R}\right. & , \infty) \| \\
& \leq \Lambda_{R}^{4-2 n}\left(\frac{\Lambda_{R}}{\Lambda_{0}}\right)^{2} P^{2 r-n} \ln \left(\frac{\Lambda_{R}}{\Lambda_{0}}\right), r+1-n \geq 0 \\
& =0, r+1-n<0
\end{aligned}
$$

where, $P^{2 r-n}$ is a polynomial of degree $2 r-n$. We do not include the proof here, for which the reader may refer to the original paper [33], but merely state that it follows by induction from the following assertions,

(i) At order $r$ in $\Lambda_{R}$,

$$
\begin{aligned}
\left\|\partial_{i_{1}, j_{1}}^{\mu_{1}} \ldots \partial_{i_{p}, j_{p}}^{\mu_{p}} A_{2 n}^{(r)}\left(p_{i}, \ldots, p_{2 n}, \Lambda\right)\right\| & \leq \Lambda^{-p} P^{2 r-n} \ln \left(\frac{\Lambda_{0}}{\Lambda_{R}}\right), r+1-n \geq 0 \\
& =0, r+1-n<0
\end{aligned}
$$

where, $A_{2 n}=\Lambda^{2 n-4} L_{2 n}$ and,

$$
\left(\frac{\partial}{\partial p_{i}^{\mu}}-\frac{\partial}{\partial p_{j}^{\mu}}\right) A_{2 n}=\partial_{i, j}^{\mu} A_{2 n}
$$


(ii) At order $r$ in $\Lambda_{R}$,

$$
\begin{aligned}
\left\|\partial_{i_{1}, j_{1}}^{\mu_{1}} \ldots \partial_{i_{p}, j_{p}}^{\mu_{p}} B_{b, 2 n}^{(r)}\left(p_{i}, \ldots, p_{2 n}, \Lambda\right)\right\| & \leq \Lambda^{-p} P^{2 r-n+1+\delta_{b 3}} \ln \left(\frac{\Lambda_{0}}{\Lambda_{R}}\right), r+2-n \geq 0 \\
& =0, r+2-n<0
\end{aligned}
$$

(iii) At order $r$ in $\Lambda_{R}$,

$$
\begin{aligned}
\left\|\partial_{i_{1}, j_{1}}^{\mu_{1}} \ldots \partial_{i_{p}, j_{p}}^{\mu_{p}} V_{2 n}^{(r)}\left(p_{i}, \ldots, p_{2 n}, \Lambda\right)\right\| & \leq \Lambda^{-p}\left(\frac{\Lambda}{\Lambda_{0}}\right)^{2} P^{2 r-n} \ln \left(\frac{\Lambda_{0}}{\Lambda_{R}}\right), r+1-n \geq 0 \\
& =0, r+1-n<0
\end{aligned}
$$

We end this part of the section by writing down the tree-level and the one loop forms for the relevant and irrelevant parts of $L$. We use (A.1) to obtain the perturbative expansion for $L_{2 n}$ order by order in $\lambda_{R}$.

Expanding the two and four point parts of $L$,

$$
\begin{aligned}
L_{2}(p,-p, \Lambda) & =\left.L_{2}(p,-p, \Lambda)\right|_{p^{2}=p_{0}^{2}}+\left.\left(p^{2}-p_{0}^{2}\right) \frac{\partial}{\partial p^{2}} L_{2}(p,-p, \Lambda)\right|_{p^{2}=p_{0}^{2}}+\Delta L_{2} \\
& =-\rho_{1}(\Lambda)-\left(p^{2}-p_{0}^{2}\right) \rho_{2}(\Lambda)+\Delta L_{2} \\
L_{4}\left(p_{i}, \Lambda\right) & =\left.L_{4}\left(p_{i}, \Lambda\right)\right|_{p_{i}=\bar{p}_{i}}+\Delta L_{4} \\
& =-\rho_{3}(\Lambda)+\Delta L_{4}
\end{aligned}
$$

Solutions for equation (A.1) with boundary conditions (4.21) for zero and one loop gives,

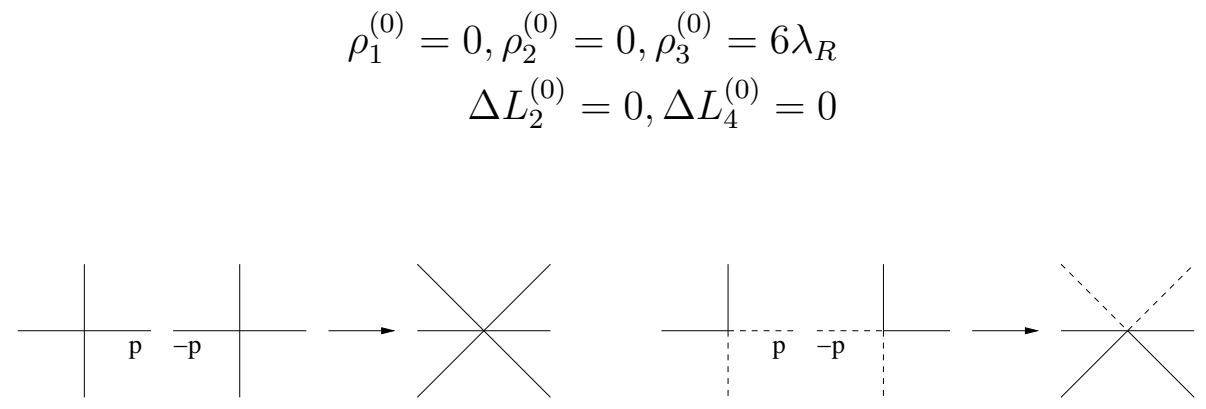

Figure 4: $L_{6}$ vertex from $L_{4}$ vertices

The $L_{6}^{(0)}$ vertex is obtained from two $L_{4}^{(0)}$ vertices as shown in Figure 4.

$$
\begin{aligned}
L_{6}^{(0)}=\int_{\Lambda}^{\Lambda_{0}} \frac{d \Lambda^{\prime}}{\Lambda^{\prime 3}} Q & \left(\Lambda^{\prime}, P\right) L_{4}^{(0)}\left(p_{1}, p_{2}, p_{3}, P, \Lambda^{\prime}\right) L_{4}^{(0)}\left(p_{4}, p_{5}, p_{6},-P, \Lambda^{\prime}\right) \\
+ & \frac{1}{2}\left(\begin{array}{l}
6 \\
3
\end{array}\right)-1 \text { permutations for all same external fields or, } \\
+ & \frac{1}{2}\left(\begin{array}{l}
4 \\
2
\end{array}\right) \text { permutations for } 2 \text { different external fields }
\end{aligned}
$$


where, $P=p_{1}+p_{2}+p_{3}$

Contracting two of the legs of the $L_{4}^{(0)}$ vertices we get the one loop $L_{2}$ amplitude, Figure 5. The weights of $N$ coming from the global $O(N)$ symmetry are indicated in brackets in the figure.
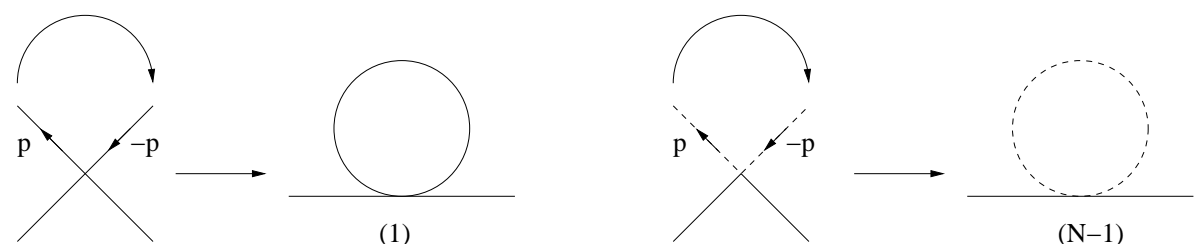

Figure 5: One loop $L_{2}$ from $L_{4}$ vertex

$$
\begin{aligned}
\rho_{1}^{(1)} & =\left.\frac{1}{2} \int \frac{d^{4} p}{(2 \pi)^{4}} \int_{\Lambda_{R}}^{\Lambda} \frac{d \Lambda^{\prime}}{\Lambda^{\prime 3}} Q\left(\Lambda^{\prime}, p\right) L_{4}^{(0)}\left(p,-p, q,-q, \Lambda^{\prime}\right)\right|_{q^{2}=p_{0}^{2}} \\
& =(N+2) \frac{\lambda_{R}}{16 \pi^{2}} \Lambda^{2}
\end{aligned}
$$

where we have only kept the $\Lambda$ dependent term.

$$
\rho_{2}^{(1)}=0, \Delta L_{2}^{(1)}=0
$$

Contracting two legs of the $L_{6}^{(0)}$ vertex, Figure 6 , we obtain the one loop $L_{4}$ amplitude. The $\Lambda$ dependent part of $\rho_{3}^{(1)}$ comes from this term only.

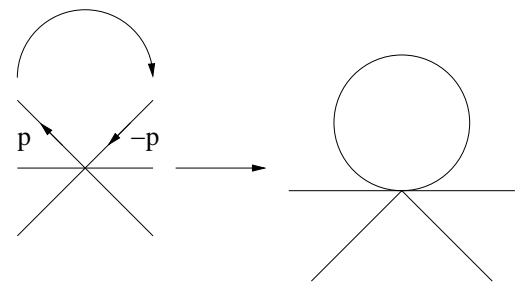

(1)

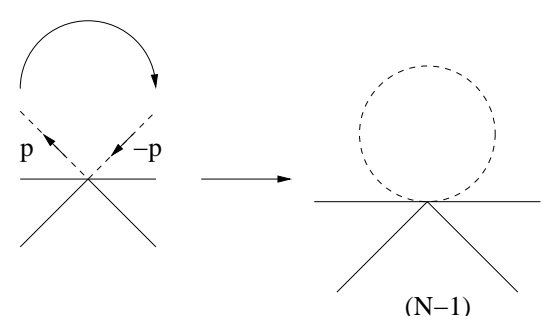

Figure 6: One loop $L_{4}$ from $L_{6}$ vertex

$$
\rho_{3}^{(1)} \sim-\left.\frac{1}{2} \int \frac{d^{4} p}{(2 \pi)^{4}} \int_{\Lambda_{R}}^{\Lambda} \frac{d \Lambda^{\prime}}{\Lambda^{\prime 3}} Q\left(p, \Lambda^{\prime}\right) L_{6}^{(0)}\left(p_{1}, p_{2}, p_{3}, p_{4}, p,-p, \Lambda^{\prime}\right)\right|_{p_{i}=\bar{p}_{i}}
$$

In the large $\Lambda$ limit,

$$
\rho_{3}^{(1)} \sim-(N+8) \frac{3 \lambda_{R}^{2}}{8 \pi^{2}} \ln \left(\frac{\Lambda^{2}}{-\mu^{2}}\right)
$$




$$
\Delta L_{4}^{(1)} \sim \frac{f\left(\bar{p}_{i}\right)}{\Lambda}
$$

$f\left(\bar{p}_{i}\right)$ is a function of external momenta. The relevant and the irrelevant operators scale at zero and one loop in the way asserted by equation (4.25) which is the primary step towards proving the renormalizability of the theory order by order in the loops by induction.

\subsection{The noncommutative case}

We have seen that the $O(N)$ symmetry of the quantum theory enables us to use the same set of RG equations for any $N$ as the $N=1$ case. We now discuss the renormalizability of the noncommutative theory to all orders in its symmetric as well as in its broken phases. Apart from the UV cutoff $\Lambda$, we shall also introduce an IR cutoff, $\Lambda_{I R}$ as mentioned earlier.

First note that the components of $L, L_{2 n}\left(p_{1}, \ldots p_{2 n}, \Lambda\right)$ contain a phase factor which accounts for the noncommutavity of the theory. This factor is of the form $e^{-\frac{i}{2} \sum_{i<j} p_{i} \wedge p_{j}}$ with all possible permutations of the external momenta. Now consider equation (4.20),

$$
\begin{aligned}
\| \int \frac{d^{4} p}{(2 \pi)^{4}} Q(p, \Lambda) L_{2 n} & \left(p_{1}, \ldots p_{2 n}, p,-p, \Lambda\right) \|+ \text { all possible permutations of } p_{i} \\
& =\left\|\int \frac{d^{4} p}{(2 \pi)^{4}} Q(p, \Lambda) e^{-\frac{i}{2} \sum_{i<j} p_{i} \wedge p_{j}}\right\| \cdot\left\|\tilde{L}_{2 n}\left(p_{1}, \ldots p_{2 n-2}, p,-p, \Lambda\right)\right\| \\
& + \text { all possible permutations of } p_{i}
\end{aligned}
$$

where $\tilde{L}_{2 n}\left(p_{1}, \ldots p_{2 n-2}, p,-p, \Lambda\right)$ is the part of $L_{2 n}$ not containing the phase factor. Equation (4.41) corresponds to the one loop vertex $L_{2 n-2}$ evaluated from the $L_{2 n}$ vertex.

Evaluating (4.41) for one particular permutation of the external momenta gives,

$$
\int \frac{d^{4} p}{(2 \pi)^{4}} Q(p, \Lambda) e^{-\frac{i}{2} \sum_{i<j} p_{i} \wedge p_{j}} e^{i\left(p \wedge \sum_{l} p_{l}\right)} \sim e^{-\frac{i}{2} \sum_{i<j} p_{i} \wedge p_{j}} \frac{\Lambda^{4}}{1+\Lambda^{2}\left(\sum_{l} p_{l}\right) o\left(\sum_{l} p_{l}\right)}(4
$$

The term on the RHS of equation (4.43) is the nonplanar term. Depending upon whether the two external momenta being contracted are consecutive to each other or not we get the usual commutative planar term or nonplanar terms respectively. See Figure 7.

The exact weights of the planar and the nonplanar graphs would not be necessary for our following discussions. We just label the weight of the planar graph by $N_{p}$. 


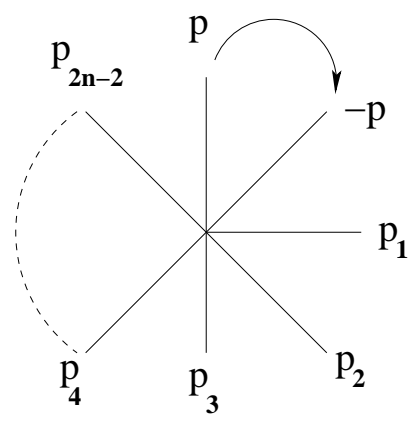

(A)

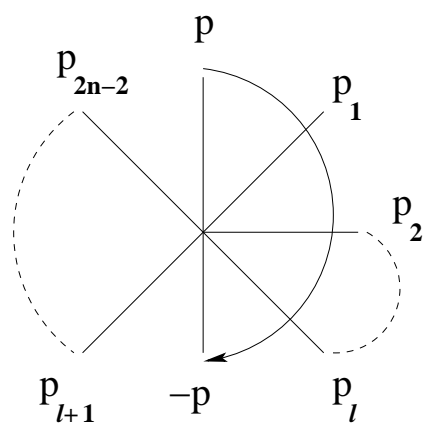

(B)

Figure 7: Contractions leading to planar and nonplanar terms are shown in (A) and (B) respectively

For all permutations of the external momenta, the scaling behavior of (4.43) with respect to $\Lambda$ is,

$$
N_{p} \Lambda^{4}+\frac{\Lambda^{4}}{1+\Lambda^{2}\left(\sum_{l} p_{l}\right) o\left(\sum_{l} p_{l}\right)}+\ldots \text { other nonplanar parts }
$$

At this point it may be noted that, with every external momentum put to zero the weight of the planar term changes due to the transmutation of the nonplanar term into the planar term.

We now turn to the question of UV renormalizability of the theory by discussing the assertions as stated in (4.25), (4.27) and (4.28). First, let us consider the case where all the external momenta are such that $\Lambda^{2}$ pop $>>1, p$ being some combination of external momenta. In this limit the nonplanar term in (4.44) scales as $\Lambda^{2}$. The scaling behavior of (4.44) is thus dominated by the planar term for large $\Lambda$. Therefore in this case the proof of assertion (i) follows exactly as the commutative case. Assuming that the assertion holds for $r=s-1$, from the RG equation (A.1), we have,

$$
\begin{aligned}
& \|\left(\Lambda \frac{\partial}{\partial \Lambda} \partial_{i_{1}, j_{1}}^{\mu_{1}} \ldots \partial_{i_{p}, j_{p}}^{\mu_{p}} L_{2 n}^{(s)}\right.\left(p_{1}, \ldots, p_{2 n}, \Lambda\right) \| \\
& \leq \Lambda^{4-2 n-p}\left[N_{p}+\frac{1}{1+\Lambda^{2}\left(\sum_{l} p_{l}\right) o\left(\sum_{l} p_{l}\right)}+\ldots\right] P^{2 s-n-1} \ln \left(\frac{\Lambda_{0}}{\Lambda_{R}}\right)
\end{aligned}
$$

For the case considered, $\Lambda^{2}$ pop $>>1$, we have,

$$
\frac{1}{1+\Lambda^{2}\left(\sum_{l} p_{l}\right) o\left(\sum_{l} p_{l}\right)} \sim \frac{1}{\Lambda^{2}\left(\sum_{l} p_{l}\right) o\left(\sum_{l} p_{l}\right)}
$$


The two powers of $\Lambda$ in the denominator, decreases the overall power of $\Lambda$ for $L_{2 n}$ in the nonplanar terms so that the overall scaling of $L_{2 n}$ with respect to $\Lambda$ for large $\Lambda$ is dominated by the planar term.

For $n \geq 3$ and $n=2, p \geq 1$, the boundary values are set to zero at $\Lambda=\Lambda_{0}$. Therefore,

$$
\begin{aligned}
\| \partial_{i_{1}, j_{1}}^{\mu_{1}} \ldots \partial_{i_{p}, j_{p}}^{\mu_{p}} L_{2 n}^{(s)} & \left(p_{1}, \ldots, p_{2 n}, \Lambda\right) \| \\
\leq & \int_{\Lambda}^{\Lambda_{0}} \frac{d \Lambda^{\prime}}{\Lambda^{\prime}}\left(\Lambda^{\prime}\right)^{4-2 n-p}\left[N_{p}+\frac{1}{\Lambda^{\prime 2}\left(\sum_{l} p_{l}\right) o\left(\sum_{l} p_{l}\right)}+\ldots\right] P^{2 s-n-1} \ln \left(\frac{\Lambda_{0}}{\Lambda_{R}}\right) \\
\leq & \Lambda^{4-2 n-p} P^{2 s-n-1} \ln \left(\frac{\Lambda_{0}}{\Lambda_{R}}\right)
\end{aligned}
$$

In general $L_{2 n+2}^{s-1}$ would have nonplanar terms as shown above. When evaluating the $L_{2 n}^{s}$ vertex from $L_{2 n+2}^{s-1}$, one has to integrate over all momenta thus including the small momentum modes. For these soft modes, $k$ one can expand the nonplanar term as,

$$
\frac{\Lambda_{e f f}(k)}{\Lambda^{2}}=\frac{1}{1+\Lambda^{2} k o k}=\left[1-\Lambda^{2} k o k+\left(\Lambda^{2} k o k\right)^{2} \ldots\right]
$$

such that $\Lambda^{2} k o k \sim O(1)$. However in this region of internal momenta, $k \sim O(1 / \theta \Lambda)$, the integral (4.43) without further exponential suppression is,

$$
e^{-\frac{i}{2} \sum_{i<j} p_{i} \wedge p_{j}} \int_{0}^{\frac{1}{\theta \Lambda}} \frac{d^{4} k}{(2 \pi)^{4}} Q(k, \Lambda)\left[1-\Lambda^{2} k o k+\left(\Lambda^{2} k o k\right)^{2} \ldots\right]
$$

The contribution from this momentum shell is suppressed by $1 / \theta \Lambda$. For larger values of $k$ the integral is again suppressed by powers of $\Lambda$ in the denominator. Therefore even though the very small momentum modes in the internal lines are included, it does not affect the cannonical scaling of the irrelevant operators. We restrict ourselves to configurations of external momenta, $p$ such that $\Lambda^{2} p o p>1$ because relevant operators scale with different coefficients from the configurations with $\Lambda^{2}$ pop $<1$ and in the continuum limit IR divergences appear when the external momenta are put to zero. It is thus clear that there is really no need for an IR cutoff, $\Lambda_{I R}$ in the loop integrals. The integrals are IR finite since we are always working with a finite UV cutoff $\Lambda$. However restriction to configurations with external momenta such that $\Lambda^{2}$ pop $>1$ implies the presence of an IR cutoff for the external momenta. Due to this reason the IR cutoff for the internal loop momenta would be formally assumed. The IR divergences in loop integrals however appear in perturbation theory. This is illustrated by an example (eqn. 4.61) at the end of this proof of UV renormalizability.

For $n=2, p=0$, the boundary values are set at $p_{i}=\bar{p}_{i}$ and $\Lambda=\Lambda_{R}$. So from (4.45), 


$$
\left\|\Lambda \frac{\partial}{\partial \Lambda} L_{4}^{(s)}\left(\bar{p}_{i}, \Lambda\right)\right\| \leq P^{2 s-3} \ln \left(\frac{\Lambda_{0}}{\Lambda_{R}}\right)
$$

which gives,

$$
\begin{aligned}
\left\|L_{4}^{(s)}\left(\bar{p}_{i}, \Lambda\right)\right\| & \leq L_{4}^{(s)}\left(\bar{p}_{i}, \Lambda_{R}\right)+P^{2 s-2} \ln \left(\frac{\Lambda_{0}}{\Lambda_{R}}\right) \\
& \leq P^{2 s-2} \ln \left(\frac{\Lambda_{0}}{\Lambda_{R}}\right)
\end{aligned}
$$

where $L_{4}^{(s)}\left(\bar{p}_{i}, \Lambda_{R}\right)$ is a constant independent of $\Lambda . L_{4}^{(s)}\left(p_{i}, \Lambda\right)$ can now be constructed for a general momentum configuration from (4.51) using the Taylor's expansion,

$$
L_{4}^{(s)}\left(p_{i}, \Lambda\right)=L_{4}^{(s)}\left(\bar{p}_{i}, \Lambda\right)+\left.\sum_{i, j=1}^{3} p_{i}^{\mu} p_{j}^{\nu} \int_{0}^{1} d \lambda(1-\lambda) \partial_{i, 4}^{\mu} \partial_{j, 4}^{\nu} L_{4}^{(s)}\left(p_{i}^{\prime}, \Lambda\right)\right|_{p_{i}^{\prime}=\lambda p}
$$

The terms on the RHS of (4.52) being bounded by (4.47) and (4.51), $L_{4}^{(s)}\left(p_{i}, \Lambda\right)$ is also bounded. The bound for the components of $L$ for $n=1$ and $p=0,2$ follows along the same line as above. This proves the assertion (i).

Let us now see the scaling behaviors of the Green's functions with respect to $\Lambda$ for a momentum configuration where some or all the external momenta are zero. As noted earlier, with each external momentum put to zero, the weights of the planar terms increases due to the transmutation of the nonplanar terms to the planar terms. Therefore although the components of $L$ scale with same power of $\Lambda$ as for the configuration with all momenta nonzero, they scale with different coefficients for the two configurations. Specifically let us take the example of $L_{4}$ where $p_{3}=p_{4}=0$. The configuration of fields for which not all external momenta are nonzero is singular and so the scaling behavior of functions for this configuration cannot be obtained from (4.52). Instead one has to define the renormalization conditions at a point where there are same number of zero external momenta so that, from (4.50) we have

$$
\begin{aligned}
\left\|L_{4}^{(s)}\left(\bar{p}_{1}, \bar{p}_{2}, 0,0, \Lambda\right)\right\| & \leq L_{4}^{(s)}\left(\bar{p}_{1}, \bar{p}_{2}, 0,0, \Lambda_{R}\right)+P^{2 s-2} \ln \left(\frac{\Lambda_{0}}{\Lambda_{R}}\right) \\
& \leq P^{2 s-2} \ln \left(\frac{\Lambda_{0}}{\Lambda_{R}}\right)
\end{aligned}
$$

The constant $L_{4}^{(s)}\left(\bar{p}_{1}, \bar{p}_{2}, 0,0, \Lambda_{R}\right)$ differs from that of (4.51), but does not affect the scaling behavior with respect to $\Lambda . L_{4}\left(p_{1}, p_{2}, 0,0, \Lambda\right)$ can now be obtained form the Taylor's expansion about $\left(\bar{p}_{1}, \bar{p}_{2}, 0,0\right)$. However due to (4.44) with $p_{3}=0, p_{4}=0$ $L_{4}^{(s)}\left(p_{1}, p_{2}, 0,0, \Lambda\right)$ scales with a different weight of $\ln \left(\frac{\Lambda}{\Lambda_{R}}\right)$ from that of (4.52). 
It is clear form these discussions that with the renormalization conditions set at nonzero external momenta the Green's functions for configurations of fields with some or all momenta zero cannot be renormalized. This was the issue in the initial sections of this paper. The bare couplings defined through the renormalization conditions for the different configurations will have different $\Lambda_{0}$ dependences. Again the different scaling of the greens funcions with respect to $\Lambda$ for these configurations plays a crucial role in studying the renormalizability of the broken phase of a spontaneously broken $O(N)$ theory. We shall discuss this at the end of this section.

The proof of assertions (ii) and (iii) follow along the same line as the commutative case and the theory is renormalizable as long as we keep away from the zero momentum limit. This concludes the proof of the renormalizability of the noncommutative $O(N)$ symmetric theory.

We now give some computations of zero and one loop contributions of the relevant and irrelevant parts of $L$ with the following boundary conditions.

$$
\begin{aligned}
& \rho_{1}\left(\Lambda_{R}, \Lambda_{0}, \rho^{0}\right)=0 \\
& \rho_{2}\left(\Lambda_{R}, \Lambda_{0}, \rho^{0}\right)=0 \\
& \rho_{3}\left(\Lambda_{R}, \Lambda_{0}, \rho^{0}\right)=2 V(\overline{\mathbf{p}}) \lambda_{R}
\end{aligned}
$$

where $V(\overline{\mathbf{p}})$ is given by $(2.3)$. The boundary values for the irrelevant couplings are set to zero at $\Lambda_{0}$ like the commutative case. As mentioned earlier the momentum integrals will now also be regulated in the IR by $\Lambda_{I R}$. However in the expressions for the one loop functions, to show the UV behavior, we drop all the $\Lambda_{I R}$ dependent terms and retain only the UV cutoff dependent pieces.

$$
\begin{gathered}
\rho_{1}^{(0)}=0, \rho_{2}^{(0)}=0, \rho_{3}^{(0)}=2 V(\overline{\mathbf{p}}) \lambda_{R} \\
\Delta L_{2}^{(0)}=0, \Delta L_{4}^{(0)}=0 \\
\rho_{1}^{(1)}=-\left.\lambda_{R} \int \frac{d^{4} p}{(2 \pi)^{4}} \int_{\Lambda_{R}}^{\Lambda} \frac{d \Lambda^{\prime}}{\Lambda^{\prime 3}} Q\left(\Lambda^{\prime}, p\right)[N+1+\cos (p \wedge q)]\right|_{q^{2}=p_{0}^{2}} \\
\sim-(N+1) \frac{\lambda_{R}}{16 \pi^{2}}\left[\Lambda^{2}+\mu^{2} \ln \left(\frac{\Lambda^{2}}{-\mu^{2}}\right)\right] \\
-\frac{\lambda_{R}}{16 \pi^{2}}\left[\Lambda_{e f f}^{2}\left(p_{0}\right)+\mu^{2} \ln \left(\frac{\Lambda_{e f f}^{2}\left(p_{0}\right)}{-\mu^{2}}\right)\right] \\
\rho_{2}^{(1)}=-\left.\frac{\partial}{\partial p^{2}} L_{2}^{(1)}(p,-p, \Lambda)\right|_{p^{2}=p_{0}^{2}} \\
\sim-\frac{\lambda_{R}}{16 \pi^{2}} \operatorname{tr}\left(\theta^{2}\right)\left[\Lambda_{e f f}^{4}\left(p_{0}\right)+\mu^{2} \Lambda_{e f f}^{2}\left(p_{0}\right)\right]
\end{gathered}
$$




$$
\begin{aligned}
\Delta L_{2}^{(1)} & =\left.\frac{\lambda_{R}}{16 \pi^{2}}\left[\Lambda_{e f f}^{2}(p)+\mu^{2} \ln \left(\frac{\Lambda_{e f f}^{2}(p)}{-\mu^{2}}\right)\right]\right|_{\Lambda} ^{\Lambda_{0}} \\
& -\left.\frac{\lambda_{R}}{16 \pi^{2}}\left[\Lambda_{e f f}^{2}\left(p_{0}\right)+\mu^{2} \ln \left(\frac{\Lambda_{e f f}^{2}\left(p_{0}\right)}{-\mu^{2}}\right)\right]\right|_{\Lambda} ^{\Lambda_{0}} \\
& +\left.\frac{\lambda_{R}}{16 \pi^{2}}\left(p^{2}-p_{0}^{2}\right) \operatorname{tr}\left(\theta^{2}\right)\left[\Lambda_{e f f}^{4}\left(p_{0}\right)+\mu^{2} \Lambda_{e f f}^{2}\left(p_{0}\right)\right]\right|_{\Lambda} ^{\Lambda_{0}} \\
& \rho_{3}^{(1)} \sim(N+3) V(\overline{\mathbf{p}}) \frac{\lambda_{R}^{2}}{4 \pi^{2}} \ln \left(\frac{\Lambda^{2}}{-\mu^{2}}\right)+G\left(\bar{p}_{i}\right)
\end{aligned}
$$

$G\left(\bar{p}_{i}\right)$ contains the nonplanar terms which in the continuum limit is only a function of the external momenta $\bar{p}_{i}$ and is divergent at low momenta.

Equations (4.55-4.60) show the scaling of the relevant and irrelevant operators with respect to $\Lambda$ at zero and one loop. As long as the external momenta are such that $\Lambda_{0}^{2}$ pop $>1$, these parts scale as asserted in (4.25). However we stress again that, ultimately one is interested in the $\Lambda_{0} \rightarrow \infty$ limit, so that these scaling behaviors persist as long as we keep away from the $p=0$ limit.

There are two points that may be noted here. For values of external momenta, $p$ such that $\Lambda_{0}^{2} p o p<<1$,

(i) One can expand $\Lambda_{0 \text { eff }}^{2}(p)$ in powers of $\Lambda_{0}^{2} p o p$, as in (4.48). The irrelevant coupling (4.59) now is dependent on the UV cutoff $\Lambda_{0}$, spoiling the usual Wilsonian picture as also noted in [17]. Thus a renormalizable noncommutative quantum theory has to be defined with an IR cutoff.

(ii) The relevant couplings scale with different coefficients from the noncommutative case where all external momenta are nonzero. They infact scale like the corresponding one loop commutative theory couplings. In the limit $\Lambda^{2} \bar{p}_{i} o \bar{p}_{j}<<1$, the would be IR divergences terms in $G\left(\bar{p}_{i}\right)$, equation (4.60) are transmuted into UV divergences and the noncommutative 4-point function scales with respect to $\ln \left(\Lambda^{2}\right)$ with the same coefficient as the commutative 4-point function (4.38).

Before discussing the renormalizability of the broken phase of the spantaneously broken theory we now take a look at an example of the two point function in perturbation theory and see the appearance of IR divergences. [8]

The contribution from the diagram, shown in Figure 8 is given by,

$$
\Gamma^{2} \sim \int d^{4} k \frac{I(k)}{\left(k^{2}+m^{2}\right)^{2}}
$$

where the nonplanar part of $I(k)$ with $n$ tadpole insertions is given by,

$$
I(k)=\frac{1}{\left(\frac{1}{\Lambda^{2}}+k o k\right)^{n}} \frac{1}{\left(k^{2}+m^{2}\right)^{n-1}}+\text { less singular terms }
$$




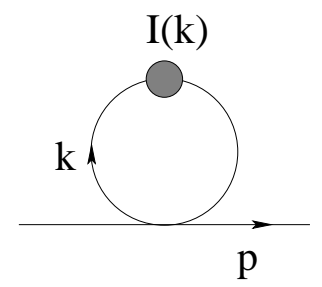

Figure 8: A higher order two-point diagram in perturbation theory. $I(k)$ contains a number of tadpole insertions

The effect of high momenta in the tadpole insertions is encoded in $\Lambda$. As all the high momenta modes are included, the singularities in $I(k)$ appears in the form of $1 /(k o k)^{n}$. The loop integral 4.61 is thus IR divergent. Note that this IR divergence in the loop integrals never comes up in the RG approach, as the loop integrals are always performed at a finite $\Lambda$.

We now turn to the issue of renormalizability of the broken phase in the spontaneously broken theory. The broken phase of the theory is defined by shifting the fields $\phi^{\alpha}$ such that,

$$
\begin{aligned}
\phi^{\alpha} & \rightarrow \sigma+v, \alpha=1 \\
& \rightarrow \pi^{\alpha}, \alpha=2, N
\end{aligned}
$$

The two and the four point contributions to the effective lagrangian of the broken phase is now given by,

$$
\begin{aligned}
& \frac{1}{2} \int d^{4} p \phi^{\alpha}(p) \phi^{\alpha}(-p) L_{2}(p,-p, \Lambda) \rightarrow \\
& \quad \frac{1}{2} \int d^{4} p\left[\sigma(p) \sigma(-p)+2 \sigma(p) v(-p)+\pi^{\alpha}(p) \pi^{\alpha}(-p)\right] L_{2}(p,-p, \Lambda)
\end{aligned}
$$

$$
\begin{aligned}
\frac{1}{4 !} \int d^{4} p_{1} d^{4} p_{2} d^{4} p_{3} d^{4} p_{4}\left[\phi^{\alpha}\left(p_{1}\right) \phi^{\alpha}\left(p_{2}\right)\right]\left[\phi^{\alpha}\left(p_{3}\right) \phi^{\alpha}\left(p_{4}\right)\right. & ] L_{4}\left(p_{1}, p_{2}, p_{3}, p_{4}, \Lambda\right) \times \\
& \times \delta^{4}\left(\sum p_{i}\right) \rightarrow
\end{aligned}
$$

$$
\begin{aligned}
\frac{1}{4 !} \int d^{4} p_{1} d^{4} p_{2} d^{4} p_{3} d^{4} p_{4}[ & {\left[\sigma\left(p_{1}\right) v\left(p_{2}\right) v\left(p_{3}\right) v\left(p_{4}\right)+\text { permutations }+\pi^{\alpha}\left(p_{1}\right) \pi^{\alpha}\left(p_{2}\right) v\left(p_{3}\right) v\left(p_{4}\right)\right.} \\
+ & \left.\left(p_{1} \rightarrow p_{3}, p_{2} \rightarrow p_{4}\right)+\ldots\right] L_{4}\left(p_{1}, p_{2}, p_{3}, p_{4}, \Lambda\right) \delta^{4}\left(\sum p_{i}\right)
\end{aligned}
$$

We have shown only these two contributions because these are the only divergent functions. The Ward identity (4.3), which is the consequence of the $O(N)$ symmetry of the symmetric phase tells that $v$ times the two point $\pi-\pi$ amplitude equals the 
$\sigma$-tadpole amplitude. This is manifest from the above two expansions. However, from the foregoing analysis we have seen that the relevant couplings scale with different coefficients whether or not the external momentum is such that $\Lambda_{0}^{2} p o p<<1$. In the $\Lambda_{0} \rightarrow \infty$ limit this inequality is satisfied for $p \rightarrow 0$. The renormalization conditions at this limit defines different $\Lambda_{0}$ dependences of the bare couplings from the renormalization conditions at $p \neq 0$.

In particular, the contributions to the $\pi-\pi$ amplitude are from,

$$
L_{2} \pi^{\alpha}(p) \pi^{\alpha}(-p)+L_{4} \pi^{\alpha}\left(p_{1}\right) \pi^{\alpha}\left(p_{2}\right) v\left(p_{3}\right) v\left(p_{4}\right)+\left(p_{1} \rightarrow p_{3}, p_{2} \rightarrow p_{4}\right)
$$

and for the $\sigma$-tadpole amplitude from,

$$
2 L_{2} \sigma(p) v(-p)+L_{4} \sigma\left(p_{1}\right) v\left(p_{2}\right) v\left(p_{3}\right) v\left(p_{4}\right)+\text { permutations }
$$

We have seen that the behavior of Green's functions with respect to $\Lambda$ changes once we put one or more external momenta to zero. The same is true for the expressions (4.66) and (4.67). Once $v$ is set to a constant at the tree level the momenta associated with $v$ are set to zero. It is clear that in this case the $L_{2}$ and $L_{4}$ functions would have different $\Lambda$ dependences from the case where $v$ is not a constant, for reasons outlined earlier. Now, since an unequal number of $v$ 's multiply (4.66) and (4.67), the weights of the UV divergent, $\Lambda$ dependent terms obtained due to transmutation of the nonplanar terms to the planar terms by setting $v$ to a constant, are different in (4.66) and (4.67). This leads to a different $\Lambda$ dependence in the $\sigma$-tadpole amplitude and the $\pi-\pi$ amplitude. Consequently these two amplitudes cannot be renormalized by the same counterterm, thus violating the Ward irentity (4.3). However the origin of the problem lies in the nonplanarity of the diagrams induced by external momenta in the loop diagrams, the resolution lies in keeping $v$ as a nonconstant background field, so that the components of (4.66) and (4.67) scale in the same way and that the Ward identity (4.3) holds. The symmetric phase of the theory has already been proved to be renormalizable as long as we have an infrared regulator. Spontaneous symmetry breaking does not affect the renormalizability and the broken phase is also renormalizable as long as $v$ is kept as a nonconstant background field.

\section{Conclusion}

We have studied the UV renormalizability of noncommutative field theories. In our discussions we have investigated this issue in the context of the $\lambda \phi^{4}$ theory, the Gross-Neveu model and the globally $O(N)$ symmetric $\phi^{4}$ model in its symmetric as well as its spontaneously broken phases. The renormalizability of the globally $O(N)$ symmetric $\phi^{4}$ theory is proved to all orders for both the symmetric as well as the broken phases with an IR cutoff. 
The zero momentum configuration for these theories is singular. Through our discussions of one loop results followed by a general analysis in the language of the Wilsonian renormalization group, the following general features of noncommutative field theories evolved.

(i) We have seen that with the renormalization conditions set at a momentum configuration where all the external momenta are nonzero, a general Green's function with some or all the external momenta zero, cannot be renormalized. With each external momentum set to zero, the weights of the UV divergent planar graphs increases due to UV/IR mixing. This leads to a scaling behavior of the greens functions with respect to the cutoff $(\Lambda)$, with a different weight than a nonzero momenta configuration. This implies that for the relevant operators, the bare couplings will have different UV cutoff dependences for these different configurations.

(ii) The different scaling of greens functions for these seperate configurations with respect to $\Lambda$ has crucial implications on the renormalizability of the spontaneously broken phase of the $O(N)$ symmetric theory. In general the renormalizability of the broken phase of the theory is unaffected by spontaneous symmetry breaking. The underlying $O(N)$ symmetry makes the broken phase renormalizable with the same number of counterterms as the symmetric phase. However in the case of noncommutative theory the one loop results indicate that this only happens when we break the symmetry by going to a vacuum which is translationally noninvariant. This can easily be understood in the language of the Wilsonian renormalization group. One of the consequences of the global $O(N)$ symmetry is the broken phase Ward identity (4.3). It can be seen from (4.66) and (4.67), keeping in mind the scaling behaviors in the foregoing discussions, that the $\sigma$-tadpole amplitude and the $\pi$ - $\pi$ amplitude would scale differently with respect to $\Lambda$ when $v$ is set to a constant. However when the constant $v$ configuration is approached as a limit of the nonconstant $v$ configuration, the Ward identity (4.3) is still preserved. We have proved this to all orders following the proof of the symmetric phase of the $O(N)$ symmetric theory.

(iii) There are no IR divergences in the loop integrals of the Renormalization group equations. This is as a consequence of always working with a finite UV cutoff $\Lambda$. IR divergences only show up when we approach a singular, zero external momentum configuration after taking the continuum limit in the solutions for the RG equations. If one keeps away from these singular field configurations, for generic external momenta, $p$, the theory is free from infrared divergences. It is because of this reason and the scaling behaviors of Green's functions in the two different momentum domains as discussed in (i), that an IR cutoff for the external momenta is necessary. IR divergences appearing in equation (4.61) are thus artifacts of perturbation theory. (iv) Finally (4.59) indicates that the irrelevant operators are badly behaved at the zero momentum configuration.

In the light of all these remarks it may be concluded that the zero momentum configuration in noncommutative seems to make sense only when it is approached as 
a limit from a nonzero momentum configuration, or in other words, the noncommutative theories are renormalizable as long as one works with an infrared cutoff.

\section{Acknowledgements}

I am indebted to B. Sathiapalan for numerous discussions and suggestions on the manuscript. I would also like to thank N. D. Haridass and H. S. Sharatchandra for valuable discussions and carefully reading the manuscript.

\section{A. RG equations for $L, B$ and $V$}

RG equation for $L$ :

$$
\begin{gathered}
\Lambda \frac{\partial}{\partial \Lambda} L_{2 n}=-\sum_{l=1}^{n}\left[\frac{Q(P, \Lambda)}{\Lambda^{2}} L_{2 l}\left(p_{1}, \ldots, p_{2 l-1}, P, \Lambda\right)\right. \\
\left.+\frac{1}{2}\left(\begin{array}{c}
2 n \\
2 l-1
\end{array}\right)-1 \text { permutations }\right] \\
-\frac{1}{2} \int \frac{d^{4} p}{(2 \pi)^{4}} \frac{Q(p, \Lambda)}{\Lambda^{2}} L_{2 n+2}\left(p_{1}, \ldots, p_{2 n}, p,-p, \Lambda\right)
\end{gathered}
$$

where, $P=\sum_{i=1}^{2 l-1} p_{i}$, such that,

$$
\begin{aligned}
\left\|\left(\Lambda^{3} \frac{\partial}{\partial \Lambda} L_{2 n}\right)\right\| \leq \sum_{l=1}^{n}\left[\frac{1}{2} D_{0}\left\|L_{2 l}(\Lambda)\right\|\right. & \left.\cdot\left\|L_{2 n+2-2 l}(\Lambda)\right\|\right] \\
& +\frac{1}{2} C \Lambda^{4}\left\|L_{2 n+2}(\Lambda)\right\|
\end{aligned}
$$

where, $D, C$ are constants independent of $\Lambda$.

RG equation for $B$ :

$$
\begin{aligned}
\left(\Lambda \frac{\partial}{\partial \Lambda}\right. & \left.+4-2 n-2 \delta_{b 1}\right) B_{b, 2 n}\left(p_{1}, \ldots, p_{2 n}\right)= \\
& -\sum_{l=1}^{n}\left[Q(P, \Lambda) A_{2 l}\left(p_{1}, \ldots, P, \Lambda\right) B_{b, 2 n+2-2 l}\left(p_{2 l}, \ldots, p_{2 n}, \Lambda\right)+\left(\begin{array}{c}
2 n \\
2 l-1
\end{array}\right)-1 \text { permutations }\right] \\
& -\frac{1}{2} \int \frac{d^{4} p}{(2 \pi \Lambda)^{4}} B_{b, 2 n+2}\left(p_{1}, \ldots, p_{2 n}, p,-p, \Lambda\right) Q(p, \Lambda) \\
& +\left.B_{1,2 n}\left(p_{1}, \ldots, p_{2 n}, \Lambda\right) \frac{1}{2} \int \frac{d^{4} q}{(2 \pi \Lambda)^{4}} B_{b, 4}(p,-p, q,-q) Q(q, \Lambda)\right|_{p^{2}=p_{0}^{2}} \\
& +\left.B_{2,2 n}\left(p_{1}, \ldots, p_{2 n}, \Lambda\right) \frac{\Lambda^{2}}{2} \int \frac{d^{4} q}{(2 \pi \Lambda)^{4}} \frac{\partial}{\partial p^{2}} B_{b, 4}(p,-p, q,-q) Q(q, \Lambda)\right|_{p^{2}=p_{0}^{2}} \\
& +\left.B_{3,2 n}\left(p_{1}, \ldots, p_{2 n}, \Lambda\right) \frac{1}{2} \int \frac{d^{4} q}{(2 \pi \Lambda)^{4}} B_{b, 6}\left(p_{i}, q,-q\right) Q(q, \Lambda)\right|_{p_{i}=\bar{p}_{i}}
\end{aligned}
$$




$$
\begin{aligned}
\|\left(\Lambda \frac{\partial}{\partial \Lambda}\right. & \left.+4-2 n-2 \delta_{b 1}\right) B_{b, 2 n}\left(p_{1}, \ldots, p_{2 n}\right) \| \\
& \leq \sum_{l=1}^{n}\left[D_{0}\left\|A_{2 l}(\Lambda)\right\| \cdot\left\|B_{b, 2 n+2-2 l}(\Lambda)\right\|\right] \\
& +\frac{1}{2} C\left\|B_{b, 2 n+2}(\Lambda)\right\|+\frac{1}{2} C\left\|B_{1,2 n}(\Lambda)\right\| \cdot\left\|B_{b, 4}(\Lambda)\right\| \\
& +\frac{1}{2} C \Lambda^{2}\left\|B_{2,2 n}(\Lambda)\right\| \cdot\left\|\partial_{1,2}^{\mu} \partial_{1,2}^{\mu} B_{b, 4}(\Lambda)\right\| \\
& +\frac{1}{2} C\left\|B_{3,2 n}(\Lambda)\right\| \cdot\left\|B_{b, 6}(\Lambda)\right\|
\end{aligned}
$$

RG equation for $V$ :

$$
\begin{aligned}
\left(\Lambda \frac{\partial}{\partial \Lambda}\right. & +4-2 n) V_{2 n}\left(p_{1}, \ldots, p_{2 n}\right)= \\
& -\sum_{l=1}^{n}\left[Q(P, \Lambda) A_{2 l}\left(p_{1}, \ldots, P, \Lambda\right) V_{2 n+2-2 l}\left(p_{2 l}, \ldots, p_{2 n}, \Lambda\right)+\left(\begin{array}{c}
2 n \\
2 l-1
\end{array}\right)-1 \text { permutations }\right] \\
& -\frac{1}{2} \int \frac{d^{4} p}{(2 \pi \Lambda)^{4}} V_{2 n+2}\left(p_{1}, \ldots, p_{2 n}, p,-p, \Lambda\right) Q(p, \Lambda) \\
& +\left.B_{1,2 n}\left(p_{1}, \ldots, p_{2 n}, \Lambda\right) \frac{1}{2} \int \frac{d^{4} q}{(2 \pi \Lambda)^{4}} V_{4}(p,-p, q,-q) Q(q, \Lambda)\right|_{p^{2}=p_{0}^{2}} \\
& +\left.B_{2,2 n}\left(p_{1}, \ldots, p_{2 n}, \Lambda\right) \frac{\Lambda^{2}}{2} \int \frac{d^{4} q}{(2 \pi \Lambda)^{4}} \frac{\partial}{\partial p^{2}} V_{4}(p,-p, q,-q) Q(q, \Lambda)\right|_{p^{2}=p_{0}^{2}} \\
& +\left.B_{3,2 n}\left(p_{1}, \ldots, p_{2 n}, \Lambda\right) \frac{1}{2} \int \frac{d^{4} q}{(2 \pi \Lambda)^{4}} V_{6}\left(p_{i}, q,-q\right) Q(q, \Lambda)\right|_{p_{i}=\bar{p}_{i}} \\
\|\left(\Lambda \frac{\partial}{\partial \Lambda}\right. & +4-2 n) V_{2 n}\left(p_{1}, \ldots, p_{2 n}\right) \| \\
& \leq \sum_{l=1}^{n}\left[D_{0}\left\|A_{2 l}(\Lambda)\right\| \cdot\left\|V_{2 n+2-2 l}(\Lambda)\right\|\right] \\
& +\frac{1}{2} C\left\|V_{2 n+2}(\Lambda)\right\|+\frac{1}{2} C\left\|B_{1,2 n}(\Lambda)\right\| \cdot\left\|V_{4}(\Lambda)\right\| \\
& +\frac{1}{2} C \Lambda^{2}\left\|B_{2,2 n}(\Lambda)\right\| \cdot\left\|\partial_{1,2}^{\mu} \partial_{1,2}^{\mu} V_{4}(\Lambda)\right\| \\
& +\frac{1}{2} C\left\|B_{3,2 n}(\Lambda)\right\| \cdot\left\|V_{6}(\Lambda)\right\|
\end{aligned}
$$

\section{References}

[1] A. Connes, M.R. Douglas and A. Schwarz, Noncommutative geometry and matrix theory: compactification on tori, J. High Energy Phys. 02(1998) 003 [hep-th/9711162]; 
A. Connes and J. Lott, Particle models and noncommutative geometry, Nucl. Phys. 18B (Proc. Suppl.) (1991) 29.

[2] A. P. Balachandran et al., Finite quantum physics and noncommutative geometry, Nucl. Phys. 37C (Proc. Suppl.) (1995) 20 hep-th/9403067.

[3] N. Seiberg and E. Witten, String Theory and noncommutative geomrtry, J. High Energy Phys. 09 (1999) 032 [hep-th/9908142].

[4] M. R. Douglas and N. Nekrasov, Noncommutative field theory Rev. Mod. Phys. 73 (2002) 977-1029, hep-th/0106048.

[5] Richard J. Szabo, Quantum Field Theory on Noncommutative Spaces, hepth/0109162

[6] I.Ya.Aref'eva, D.M.Belov, A.A.Giryavets, A.S.Koshelev and P.B.Medvedev, Noncommutative Field Theories and (Super)String Field Theories, hep-th/0111208]

[7] T. Filk, Divergences in a field theory on quantum space, Phys. Lett. B 376 (1996) 53.

[8] S. Minwalla, M. V. Raamsdonk and N. Seiberg, Noncommutative perturbatibe dynamics, J. High Energy Phys. 02 (2000) 020 hep-th9912072

M. V. Ramsdonk, N. Seiberg, Comments on noncommutative perturbative dynamics, J. High Energy Phys. 03 (2000) 035 hep-th/0002186.

[9] N. Seiberg, L. Susskind and N. Toumbas, Space/time noncommutavity and causality, J. High Energy Phys. 06 (2000) 044 hep-th/0005015.

[10] J. Gomis and T. Mehen, Space-time noncommutative field theories and unitarity, Nucl. Phys. B 591 (2000) 265 hep-th/0005129

L. Alvarez-Gaume, J. L. F. Barbon, R. Zwicky, Remarks on time-space noncommutative field theories, J. High Energy Phys. 05 (2001) 057 hep-th/0103069

[11] E. T. Akhmedov, P. DeBoer and G. W. Semenoff, Running couplings and triviality of field theories on non-commutative spaces, Phys. Rev D64 (2001) 065005; Noncommutative Gross-Neveu model at large N, J. High Energy Phys. 06 (2001) 009 hep-th/0103199

H. O. Girotti, M. Gomes, V. O. Rivelles and A. J. da Silva, The noncommutative supersymmetric nonlinear sigma model, hep-th/0102101.

[12] B. A. Campbell and K. Kaminsky, Noncommutative field theory and spontaneous symmetry breaking, Nucl. Phys B 581 (2000) 240 hep-th/0003137; Noncommutative linear sigma models Nucl. Phys. B 606 (2001) 613-635 hep-th/0102022.

[13] F. J. Petriello, The Higgs mechanism in noncommutative gauge theories, Nucl. Phys. B 601 (2001) 169 hep-th/0101109.

[14] S. Vaidya, Perturbative dynamics on fuzzy $S^{2}$ and $R P^{2}$, Phys. Lett. $\mathbf{B 5 1 2}$ (2001) 403-411 hep-th/0102212 
[15] S. Sarkar and B. Sathiapalan, Comments on the renormalizability of the broken phase in noncommutative scalar field theory, J. High Energy Phys. 05 (2001) 049 hepth/0104106.

[16] S. S. Gubser and S. L. Sondhi, Phase structure of noncommutative scalar field theories, Nucl. Phys B605 (2001) 395-424 [hep-th/0006119].

[17] L. Griguolo and M. Pietroni, Wilsonian renormalization group and the noncommutative IR/UV connection, J. High Energy Phys.05 (2001) 032 [hep-th/0104217]

[18] W. H. Huang, Two-loop effective potential in noncommutative scalar field theory, Phys. Lett. B 496 (2000) 206 hep-th/0009067.

[19] I. Y. Aref'eva, D. M. Belov and A. S. Koshelev, Two-loop diagrams in noncommutative $\phi^{4}$ theory, Phys. Lett. B 476 (2000) 431 [hep-th/9912075];

A. Micu, M. M. Sheik-Jabbari Noncommutative $\phi^{4}$ theory at two loops, J. High Energy Phys. 01 (2001) 025 hep-th/0008057.

[20] I. Chepelev and R. Roiban, Renormalization of quantum field theories on noncommuatative $R^{d}$, 1. Scalars, J. High Energy Phys. 05 (2000) 037 hep-th/9911098; Convergence theorem for non-commutative Feynman graphs and renormalization, J. High Energy Phys. 03 (2001) 001 hep-th/0009090.

[21] H. O. Girotti, M. Gomes, V. O. Rivelles and A. J. da Silva A consistent noncommutative field theory: the Wess-Zumino model, Nucl Phys. B 587(2000) 299 hepth/0005272.

[22] I. Y. Aref'eva, D. M. Belov and A. S. Koshelev, A note on UV/IR for noncommutative complex scalar field, hep-th/0001215; UV/IR mixing for noncommutative complex scalar field theory. II: interaction with gauge fields, hep-th/0003176].

[23] A. Matusis, L. Susskind and N. Toumbas, the IR/UV connection in the noncommutative gauge theories, J. High Energy Phys. 12 (2000) 002 hep-th/0002075.

[24] C. P. Martin and D. Sanchez-Ruiz, The one-loop UV divergent structure of U(1) Yang Mills theory on noncommutative $R^{4}$, Phys. Rev. Lett. 83 (1999) 476 hep-th/9903077 M. Hayakawa, Perturbative analysis on infrared and ultraviolet aspects of noncommutative $Q E D$ on $R^{4}$, hep-th/9912167

F. Ruiz Ruiz, Gauge-fixing independence of IR divergences in non-commutative $U(1)$, perturbative tachyonic instabilities and supersymmetry, Phys. Lett. B 502 (2001) 274-278 hep-th/0012171

Y. Liao One loop renormalization of spontaneously broken U(2) gauge theory on noncommutative spacetime, J. High Energy Phys. 11 (2001) 067 [ hep-th/0110112]; One loop renormalizability of spontaneously broken gauge theory with a product of gauge groups on Noncommutative Spacetime: the $U(1) \times U(1)$ Case hep-th/0201135.

[25] A. Armoni, Comments on perturbative dynamics of non-commutative Yang-Mills theory, Nucl. Phys. B 593 (2001) 229-242 hep-th/0005208 
V. V. Khoze and G Travaglini, Wilsonian effective actions and IR/UV mixing in noncommutative gauge theories, J. High Energy Phys.01 (2001) 026 hep-th/0011218]

L.Bonora, M.Salizzoni Renormalization of noncommutative $U(N)$ gauge theories Phys. Lett. B 504 (2001) 80-88 [hep-th/0011088]

A. Zanon, $N=1$,2 super $U(N)$ Yang-Mills: $U V / I R$ mixing and effective action results at one loop, Phys Lett. B 502 (2001) 265 hep-th/0012009

A. Armoni, R. Minasian, S. Theisen On non-commutative N=2 super Yang-Mills, Phys. Lett. B513 (2001) 406-412 hep-th/0102007].

[26] N. Ishibashi, S. Iso, H. Kawai, Y. Kitazawa Wilson Loops in Noncommutative Yang Mills , Nucl. Phys. B573 (2000) 573-593 hep-th/9910004

D. J. Gross, A. Hashimoto, N. Itzhaki Observables of non-commutative gauge theories, Adv. Theor. Math. Phys. 4 (2000) 893-928 hep-th/0008075]

A. Dhar, S. R. Wadia A note on gauge invariant operators in noncommutative gauge theories and the Matrix Model, Phys. Lett. B 495 (2000) 413-417 hep-th/0008144.

H. Liu *-Trek II: $*_{n}$ Operations, Open Wilson Lines and the Seiberg-Witten Map, Nucl. Phys. B 614 (2001) 305-329 hep-th/0011125

M. Rozali, M. V. Raamsdonk Gauge Invariant Correlators in Non-Commutative Gauge Theory, Nucl. Phys. B 608 (2001) 103-124 hep-th/0012065.

[27] R. Gopakumar, S. Minwalla, A. Strominger Noncommutative Solitons, J. High Energy Phys. 05 (2000) 020 hep-th/0003160

G. Mandal, S-J. Rey, S. Wadia Quantum aspects of GMS solutions of noncommutative field theory and large $N$ limit of Matrix Models hep-th/0111059.

[28] Y. Kiem, S.-J. Rey, H.-T. Sato, J.-T. Yee Open Wilson Lines and Generalized Star Product in Noncommutative Scalar Field Theories, Phys. Rev. bf D 65 (2002) 026002 hep-th/0106121

Y. Kiem, S. Lee, S.-J. Rey, H.-T. Sato it Interacting Open Wilson Lines in Noncommutative Field Theories, Phys. Rev. D 65 (2002) 046003 hep-th/0110215.

[29] Y. Kinar, G. Lifschytz and J. Sonnenschein, UV/IR connection, a matrix perspective, J. High Energy Phys. 08 (2001) 001 hep-th/0105089.

[30] M. V. Raamsdonk, The Meaning of Infrared Singularities in Noncommutative Gauge Theories , J. High Energy Phys. bf 11 (2001) 006 hep-th/0110093.

A. Armoni, E. Lopez UV/IR Mixing via Closed Strings and Tachyonic Instabilities hep-th/0110113.

[31] F. Ruiz Ruiz, UV/IR mixing and the Goldstone theorem in noncommutative field theory, hep-th/0202011

[32] H.S. Sharatchandra, A renormalization group analysis of local quantum field theory, Unpublished, TIFR Preprint, TIFR-TH-79-36 (1979)

[33] J. Polchinski, Renormalization and effective lagrangians, Nucl. Phys. B231 (1984) 269-295 
[34] S. Coleman and E. Weinberg, Radiative corrections as origin of spontaneous symmetry breaking, Phys. Rev. D7 (1973) 1888-1910

[35] D. J. Gross and A. Neveu, Dynamical symmetry breaking in asymptotically free field theories, Phys. Rev. D10 (1974) 3235. 